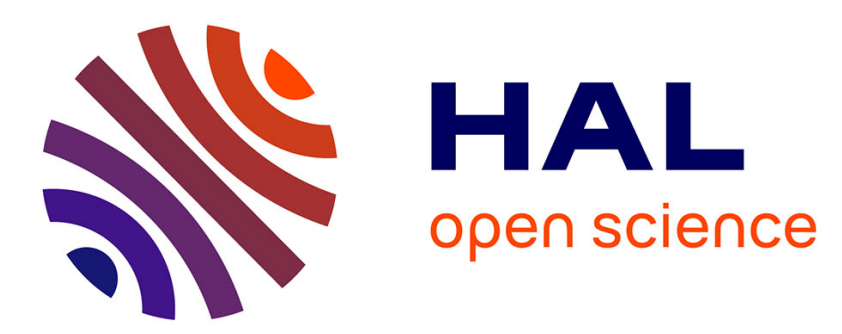

\title{
Une approche parallèle et multi-échelles en calcul des structures : exemples et performances
}

David Dureisseix, Pierre Ladevèze

\section{To cite this version:}

David Dureisseix, Pierre Ladevèze. Une approche parallèle et multi-échelles en calcul des structures: exemples et performances. Revue Européenne des Éléments Finis, 1998, 7 (1-3), pp.73-87. 10.1080/12506559.1998.11690466 . hal-00325941

\section{HAL Id: hal-00325941 \\ https://hal.science/hal-00325941}

Submitted on 7 Dec 2017

HAL is a multi-disciplinary open access archive for the deposit and dissemination of scientific research documents, whether they are published or not. The documents may come from teaching and research institutions in France or abroad, or from public or private research centers.
L'archive ouverte pluridisciplinaire HAL, est destinée au dépôt et à la diffusion de documents scientifiques de niveau recherche, publiés ou non, émanant des établissements d'enseignement et de recherche français ou étrangers, des laboratoires publics ou privés. 


\title{
Une approche parallèle et multi-échelles en calcul des structures : exemples et performances
}

\author{
David Dureisseix ${ }^{1}$ and Pierre Ladevèze ${ }^{1}$ \\ ${ }^{1}$ Laboratoire de Mécanique et Technologie, (E.N.S. de \\ Cachan / Université Paris 6 / C.N.R.S.), 61 Avenue du \\ Président Wilson, 94235 CACHAN CEDEX, FRANCE
}

Parmi les méthodes de calcul de structures bien adaptées aux ordinateurs à architecture parallèle, que ce soit à mémoire distribuée ou partagée, les méthodes de décomposition de domaine peuvent être classées en approches primales, duales ou "mixtes", comme celle qui est utilisée ici : les inconnues sont à la fois les déplacements et efforts aux interfaces. Un inconvénient de ces méthodes est la diminution du taux de convergence lorsque le nombre de sous-domaines croît. L'utilisation d'un mécanisme global de propagation d'information parmi tous les sous-domaines peut y remédier. Nous proposons ici de prendre en compte deux échelles introduites lors d'une décomposition en sous-structures et interfaces. Le problème à grande échelle est alors utilisé pour permettre l'échange global d'information et ainsi améliorer les performances. De plus, nous comparons cette approche à différentes autres méthodes de décomposition de domaine, dont plusieurs variantes de la méthode FETI.

In structural analysis field, domain decomposition methods conducts to algorithms well suited to parallel architecture computers. Several approaches related with these methods have been designed; for instance, primal approaches, dual ones or "mixed" ones, as the approach used herein : both displacements and efforts onto interfaces are the unknowns. A general drawback for these methods is the decrease of convergence when increasing the number of substructures. Using a global mechanism to propagate information among all the substructures can avoid this drawback. We are proposing herein to take into account two scales introduced when decomposing the structure into substructures and interfaces. The large scale pro- 
blem is then used to build a global exchange of information and therefore to improve performance. Moreover, comparisons with other decomposition methods, and in particular with several variants of the FETI method are proposed.

This is a preprint of the article published by Taylor \& Francis in Revue Européenne des Éléments Finis / European Journal of Computational Mechanics 7(1-3) :73-87, 1998, available online : http://www.tandfonline. com/10.1080/12506559.1998.11690466

Keywords : décomposition de domaine, approche non-incrémentale, multiéchelles

domain decomposition, non-incremental approach, multi-level.

\section{Introduction}

Les ordinateurs les plus performants sont aujourd'hui construits autour d'une architecture parallèle 19. Pour utiliser de telles architectures, la construction d'algorithmes adaptés n'est pas immédiate et de nombreuses recherches sont en cours pour bâtir des algorithmes "parallèles".

Pour le calcul de structures, les techniques de décomposition de domaine sont bien adaptées à ces multi-processeurs, avec une mémoire partagée ou distribuée, 23. Parmi ces techniques, on peut distinguer les méthodes directes où un opérateur condensé sur l'ensemble des interfaces est explicitement construit, comme les méthodes multi-frontales $(4,7])$, et les méthodes itératives, souvent basées sur un algorithme de type gradient conjugué. Pour ces dernières, et dans le cas où la décomposition en sousdomaines se fait sans recouvrement, l'accent peut être porté sur la continuité du déplacement aux interfaces, comme pour la méthode de Schur primale ([16, par exemple), ou sur l'équilibre des efforts aux interfaces, comme pour la méthode de Schur duale ([9, 10]).

L'approche qui sert de base à cette étude, est construite à partir d'une technique de sous-structuration et de l'approche à grand incrément de temps (LATIN). La structure est décomposée en sous-structures et interfaces, et ces dernières jouent un rôle majeur pour cette approche. En particulier, efforts et déplacements aux interfaces sont traités à égalité; c'est pourquoi, il s'agit ici d'une approche "mixte" en décomposition de domaine. Ses principes sont décrits dans [14, sa faisabilité a été montrée dans [15], et [3], 2] proposent des exemples significatifs lorsque les sousstructures ont un comportement élastique linéaire.

Sur cette décomposition en sous-structures et interfaces, nous introduisons une approche parallèle et multi-échelles où le problème à grande échelle est utilisé pour permettre un échange global et donc rapide d'informations. Les approches LATIN ont été initialement développées pour les problèmes non linéaires d'évolution, aussi l'écriture de l'approche parallèle, qui est 
l'objet de ce travail, ne poserait pas de difficultés dans un tel cadre. Toutefois, pour simplifier la présentation et donner les idées forces, nous nous restreignons ici au cas de l'élasticité linéaire. Dans ce cas, le temps n'intervient plus et seule la configuration finale nous intéresse.

L'approche ainsi proposée est ensuite comparée à d'autres techniques de décomposition de domaine, en particulier à plusieurs versions de la méthode FETI.

\section{Formulation du problème}

Dans le but de procéder au traitement en parallèle du problème, la structure est décomposée en sous-structures $\Omega^{E}, E \in \mathbf{E}$, et en interfaces $L^{E E^{\prime}}$. Chacune est une entité mécanique à part entière avec ses propres variables et ses propres équations.

\subsection{Comportement des sous-structures}

Chaque sous-structure $\Omega^{E}$ est soumise à l'action de son environnement (les interfaces voisines) : une répartition d'efforts $\underline{F}^{E}$ et un champ de déplacement $\underline{W}^{E}$ sur sa frontière $\partial \Omega^{E}$. Éventuellement, $\underline{f}_{d}$ est une densité de charge volumique imposée (figure 11. Pour tout $E \in \mathbf{E},\left(\underline{W}^{E} ; \underline{F}^{E}\right)$ doit vérifier :

- les équations de liaison :

$$
\exists \underline{U}^{E} \in \mathcal{U}^{E}, \quad \varepsilon^{E}=\varepsilon\left(\underline{U}^{E}\right) \quad \text { et } \quad \underline{U}_{\mid \partial \Omega^{E}}^{E}=\underline{W}^{E}
$$

où $\mathcal{U}^{E}$ est l'ensemble des champs de déplacement définis sur $\Omega^{E}$ qui possèdent une énergie finie, et où $\varepsilon^{E}$ est la déformation associée.

- les équations d'équilibre:

il existe un champ de contraintes $\sigma^{E}$ qui équilibre $\underline{f}_{d}$ et $\underline{F}^{E}$, i.e.

$$
\begin{aligned}
& \forall \underline{U}^{\star} \in \mathcal{U}^{E}, \\
& \quad \int_{\Omega^{E}} \operatorname{Tr}\left[\boldsymbol{\sigma}^{E} \varepsilon\left(\underline{U}^{\star}\right)\right] d \Omega=\int_{\Omega^{E}} \underline{f}_{d} \cdot \underline{U}^{\star} d S+\int_{\partial \Omega^{E}} \underline{F}^{E} \cdot \underline{U}_{\mid \partial \Omega^{E}}^{\star} d S
\end{aligned}
$$

- la relation de comportement :

ici, le comportement est celui de l'élasticité linéaire (K est le tenseur de Hooke) et

$$
\boldsymbol{\sigma}^{E}=\mathbf{K} \varepsilon^{E}
$$

L'ensemble des inconnues $\left(\underline{W}^{E}, \underline{F}^{E}, \underline{U}^{E}, \boldsymbol{\sigma}^{E}\right)$, pour $E \in \mathbf{E}$, qui caractérisent l'état des sous-structures, est noté $\mathbf{s}$. 

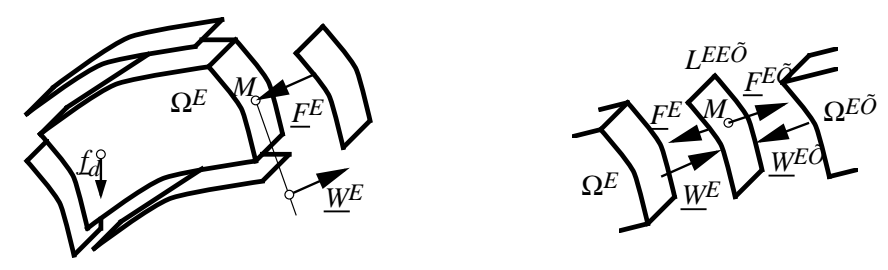

FiguRE 1: Sous-structure et interface

\subsection{Comportement des interfaces}

L'état de la liaison entre deux sous-structures $\Omega^{E}$ et $\Omega^{E^{\prime}}$ est défini à partir des déplacements et des efforts $\left(\underline{W}^{E} ; \underline{F}^{E}\right)$ et $\left(\underline{W}^{E^{\prime}} ; \underline{F}^{E^{\prime}}\right)$ (figure 1). Par exemple, pour une liaison parfaite, ils doivent vérifier :

$$
\underline{F}^{E}+\underline{F}^{E^{\prime}}=0 \quad \text { et } \quad \underline{W}^{E}=\underline{W}^{E^{\prime}}
$$

D'autres types de liaison peuvent être envisagés : liaisons à effort imposé, à déplacement imposé, liaisons de contact unilatéral avec ou sans frottement..., 14, 2]. Nous ne considèrerons ici que le cas des interfaces parfaites.

\subsection{Description de l'algorithme mono-échelle}

La structure est maintenant considérée comme un assemblage de sousstructures et d'interfaces. Les inconnues du problème sont à la fois les déplacements et les efforts sur les interfaces ; c'est pourquoi l'approche utilisée est qualifiée de mixte.

En suivant la trame des approches à grand incrément de temps (LATIN en anglais) [15, les équations sont réparties en deux groupes pour séparer les difficultés :

- $\boldsymbol{\Gamma}$ est l'ensemble des inconnues $\mathbf{s}$ qui vérifient le comportement de chaque interface [4]

- $\mathbf{A}_{\mathbf{d}}$ est celui des $\mathbf{s}$ qui vérifient le comportement de chaque sousstructure 1, 2, 3.

La solution cherchée $\mathbf{s}_{e x}$ est alors l'intersection de $\mathbf{A}_{\mathbf{d}}$ et $\boldsymbol{\Gamma}$. Un algorithme à deux étapes construit alors successivement un élément de $\mathbf{A}_{\mathbf{d}}$ puis de $\boldsymbol{\Gamma}$ (figure 2). Chaque étape nécessite une direction de recherche; elles sont les paramètres de la méthode :

- l'étape locale utilise la direction de recherche $\mathbf{E}^{+}$:

$$
(\underline{\hat{F}}-\underline{F})-k(\underline{\hat{W}}-\underline{W})=0
$$

Trouver $\hat{\mathbf{s}} \in \mathbf{\Gamma}$ de façon à ce que $\hat{\mathbf{s}}-\mathbf{s}_{n}$ appartienne à la direction de recherche $\mathbf{E}^{+}$est un problème local sur les interfaces. Il est trivialement parallélisable. 
- l'étape linéaire utilise la direction de recherche $\mathbf{E}^{-}$:

$$
(\underline{F}-\underline{\hat{F}})+k(\underline{W}-\underline{\hat{W}})=0
$$

Le problème consiste alors à trouver $\mathbf{s}_{n+1} \in \mathbf{A}_{\mathbf{d}}$ de telle façon à ce que $\mathbf{s}_{n+1}-\hat{\mathbf{s}}$ appartienne à $\mathbf{E}^{-}$. Cela conduit à un problème global uniquement par sous-structure, qui peut donc être traité en parallèle une fois les sous-structures distribuées parmi les processeurs disponibles, de la même manière que leurs interfaces voisines.

Un indicateur de convergence peut ainsi être bâti à partir de $\left\|\hat{\mathbf{s}}-\mathbf{s}_{n}\right\|$.

Dans le cas de l'élasticité linéaire, cet algorithme est similaire à celui proposé dans [13, [17, 11]. D'un point de vue "milieu continu", il converge vers la solution du problème de référence sans décomposition. Des conditions précises de convergence peuvent être trouvées dans 14 .

\section{Une extension multi-échelles}

Un comportement classique des méthodes de type décomposition de domaine est la dégradation du taux de convergence lorsque le nombre de sous-structures croît [1], 21]. Par exemple, pour le problème modèle d'une structure bidimensionnelle élancée soumise à un chargement de flexion parabolique (figure 3), la solution de référence $(\underline{U} ; \boldsymbol{\sigma})_{\text {ref éléments finis permet }}$ de définir le taux de convergence relatif à l'erreur en énergie :

$$
\tau=-\log \frac{e_{n+1}}{e_{n}} \quad \text { où } \quad e_{n}^{2}=\frac{\frac{1}{2} \int_{\Omega} \operatorname{Tr}\left[\left(\boldsymbol{\sigma}_{n}-\boldsymbol{\sigma}_{\mathrm{ref}}\right) \mathbf{K}^{-1}\left(\boldsymbol{\sigma}_{n}-\boldsymbol{\sigma}_{\mathrm{ref}}\right)\right] d \Omega}{\frac{1}{2} \int_{\Omega} \operatorname{Tr}\left[\boldsymbol{\sigma}_{\mathrm{ref}} \mathbf{K}^{-1} \boldsymbol{\sigma}_{\mathrm{ref}}\right] d \Omega}
$$

Le paramètre de direction de recherche peut être ici choisi sous la forme $k=E / L_{0}, E$ étant le module d'Young du matériau et $L_{0}$ une longueur qui caractérise donc la direction de recherche. Pour l'approche LATIN monoéchelle précédente, la valeur "optimale" de $L_{0}$ est liée à la taille de la structure, [3]. La figure 4 reporte alors le taux de convergence moyen pour atteindre une convergence poussée $\left(e_{n} \leq 0.1 \%\right)$ en fonction du nombre de sous-structures. Il se dégrade effectivement lorsque le nombre de sousstructures croît. Une interprétation possible est de noter qu'à chaque itération de l'algorithme, l'information ne se propage que d'une couche de sousstructures à la fois et non pas globalement dans l'assemblage de sousstructures et d'interfaces.

Pour remédier à un tel inconvénient un mécanisme d'échange global d'information parmi toutes les sous-structures est en général efficace. Plusieurs algorithmes de décomposition de domaine en utilisent aujourd'hui un, comme la méthode FETI, [10], ou la "Balancing Domain Decomposition method", [18. Pour construire ce mécanisme, nous choisissons ici de 
représenter la solution cherchée sur différentes échelles :

$$
\left(\underline{U}^{E} ; \boldsymbol{\sigma}^{E}\right)=\left(\underline{U}_{1}^{E} ; \boldsymbol{\sigma}_{1}^{E}\right)+\left(\underline{U}_{2}^{E} ; \boldsymbol{\sigma}_{2}^{E}\right)
$$

où 1 et 2 sont respectivement utilisés pour les inconnues relatives à la grande échelle (grandeurs effectives) et aux corrections sur l'échelle fine. On choisit ici de conserver le problème sur la grande échelle global. Il va ainsi jouer le rôle de mécanisme d'échange d'information parmi les sousstructures. L'échelle fine est quant à elle traitée par la technique précédente de sous-structuration.

Chaque échelle peut provenir d'une modélisation différente de la structure, par exemple ici, de 2 maillages différents avec éléments emboîtés. Elles seront notée par la suite $\Omega_{1}$ et $\Omega_{2}$. Les principes de cette technique ont été décrits dans [6]. Les transferts d'information entre les échelles sont alors réalisés avec un opérateur de prolongation $\mathbb{P}$ et un opérateur de restriction $\mathbb{R}=\mathbb{P}^{T}$, suivant la terminologie des méthodes multigrilles, [12], 20]. La transposition est définie avec la forme symétrique en énergie :

$$
\sum_{E \in \mathbf{E}} \int_{\Omega_{2}^{E}} \operatorname{Tr}\left[\boldsymbol{\varepsilon}\left(\mathbb{P}^{E} \underline{\underline{U}}\right) \boldsymbol{\sigma}^{E}\right] d \Omega=\int_{\Omega_{1}^{E}} \operatorname{Tr}\left[\boldsymbol{\varepsilon}(\overline{\bar{U}}) \sum_{E \in \mathbf{E}} \mathbb{R}^{E} \boldsymbol{\sigma}^{E}\right] d \Omega
$$

$(\underline{\underline{U}}, \overline{\boldsymbol{\sigma}})$ est la solution effective, c'est-à-dire la partie de la solution définie sur le maillage de $\Omega_{1}$ (alors $\underline{U}_{1}^{E}=\mathbb{P}^{E} \underline{\bar{U}}$ et $\overline{\boldsymbol{\sigma}}=\sum_{E \in \mathbf{E}} \mathbb{R}^{E} \boldsymbol{\sigma}_{1}^{E}$ ). Avec des maillages emboités, la prolongation peut être réalisée avec une projection de type éléments finis hiérarchiques, [24].

L'équilibre peut maintenant être écrit sur chaque échelle :

- sur l'échelle fine 2 , sur chaque sous-structure $\Omega_{2}^{E}$,

$$
\begin{aligned}
\forall \underline{U}_{2}^{\star} \in \mathcal{U}_{2}^{E}, \quad \int_{\Omega_{2}^{E}} \operatorname{Tr}\left[\boldsymbol{\sigma}_{2}^{E} \varepsilon\left(\underline{U}_{2}^{\star}\right)\right] d \Omega & =\int_{\Omega_{2}^{E}} \underline{f}_{d} \cdot \underline{U}_{2}^{\star} d \Omega+\int_{\partial \Omega_{2}^{E}} \underline{F}_{2}^{E} \cdot \underline{U}_{2}^{\star} d S+ \\
& -\int_{\Omega_{2}^{E}} \operatorname{Tr}\left[\varepsilon\left(\underline{U}_{1}\right) \mathbf{K} \varepsilon\left(\underline{U}_{2}^{\star}\right)\right] d \Omega
\end{aligned}
$$

$\mathcal{U}_{2}^{E}$ est la partie de $\mathcal{U}^{E}$ correspondant à l'échelle fine 2, vérifiant des conditions aux limites homogènes. Sur l'échelle fine, le champ de contraintes $\boldsymbol{\sigma}_{2}^{E}$ doit aussi équilibrer $-\boldsymbol{\sigma}_{1}^{E}=-\mathbf{K} \varepsilon\left(\underline{U}_{1 \mid \Omega^{E}}\right)=-\mathbf{K} \varepsilon\left(\mathbb{P}^{E} \underline{\underline{U}}\right)$. En utilisant la direction de recherche $\left(\underline{F}_{2}-\underline{\hat{F}}_{2}\right)+k\left(\underline{W}_{2}-\underline{\hat{W}}_{2}\right)=0$, avec $\underline{W}_{2}^{E}=\underline{U}_{2}^{E}{ }_{\mid \partial \Omega^{E}}$, la formulation en déplacement discrétisée de ce problème est

$$
\left(\left[K^{E}\right]+\left[k^{E}\right]\right)\left[U_{2}^{E}\right]=\left[f_{d}^{E}\right]+\left[\hat{f}^{E}\right]-\left[B \boldsymbol{\sigma}_{1}^{E}\right]
$$

où $\left[K^{E}\right]$ et $\left[k^{E}\right]$ sont des rigidités constantes au cours des itérations, provenant du matériau et de la direction de recherche, $[3],\left[\hat{f}^{E}\right]$ est un terme de charge dû à $\underline{\hat{F}}^{E}{ }_{2}+k \underline{\hat{W}}^{E}$, et $B$ l'opérateur qui donne les forces généralisées équilibrant un champ de contrainte. 
- sur la grande échelle 1,

$$
\begin{aligned}
\forall \underline{U}_{1}^{\star} \in \mathcal{U}_{1}, \quad \int_{\Omega_{1}} \operatorname{Tr}\left[\boldsymbol{\sigma}_{1} \varepsilon\left(\underline{U}_{1}^{\star}\right)\right] d \Omega & =\int_{\Omega_{1}} \underline{f}_{d} \cdot \underline{U}_{1}^{\star} d \Omega+ \\
& -\int_{\Omega_{1}} \operatorname{Tr}\left[\varepsilon\left(\underline{U}_{1}^{\star}\right) \sum_{E \in \mathbf{E}} \mathbf{K} \varepsilon\left(\underline{U}_{2}^{E}\right)\right] d \Omega
\end{aligned}
$$

$\mathcal{U}_{1}$ est relatif aux champs définis sur l'ensemble de la structure $\Omega$, vérifiant des conditions aux limites homogènes. On peut alors remarquer qu'il n'y a pas séparation des échelles : sur la grande échelle, le champ de contraintes $\overline{\boldsymbol{\sigma}}$ doit aussi équilibrer $-\overline{\boldsymbol{\sigma}}_{2}=-\sum_{E \in \mathbf{E}} \overline{\boldsymbol{\sigma}}_{2 \mid \Omega^{E}}=$ $-\sum_{E \in \mathbf{E}} \mathbb{R}^{E} \mathbf{K} \varepsilon\left(\underline{U}_{2}^{E}\right)$.

Toujours après discrétisation de la formulation en déplacement, on obtient

$$
\left[K_{1}\right]\left[U_{1}\right]=\left[B \overline{\boldsymbol{\sigma}}_{d}\right]-\left[B \overline{\boldsymbol{\sigma}}_{2}\right]
$$

où $\overline{\boldsymbol{\sigma}}_{d}$ provient des charges extérieures :

$$
\forall \underline{U}_{1}^{\star} \in \mathcal{U}_{1}, \quad \int_{\Omega_{1}} \underline{f}_{d} \cdot \underline{U}_{1}^{\star} d \Omega=\int_{\Omega_{1}} \operatorname{Tr}\left[\overline{\boldsymbol{\sigma}}_{d} \varepsilon\left(\underline{U}_{1}^{\star}\right)\right] d \Omega
$$

La solution est alors recherchée successivement dans les deux échelles à chaque itération de la méthode LATIN sur l'échelle fine sous-structurée, [5]. La table 1 décrit cet algorithme. Les approches LATIN ont été implantées dans le code semi-industriel CASTEM2000 développé au CEA de Saclay, 22 .

Pour l'exemple précédent, le gain en taux de convergence est illustré sur la figure 4 quand chaque sous-structure est maillée avec 2048 éléments triangulaires isoparamétriques à 6 nœuds, et ceci pour chaque sous-structuration (4, 16 et 64 sous-structures). La grande échelle a été maillée avec un nombre d'éléments triangulaires à 3 nœuds égal à deux fois le nombre de sousstructures. Le problème de référence possède respectivement 33 400, 132354 et 526850 degrés de liberté.

La quasi-indépendance du taux de convergence vis à vis du nombre de sous-structures illustre l'extensibilité numérique de l'approche LATIN multi-échelles.

On peut aussi noter sur cet exemple que la nouvelle valeur optimale pour la direction de recherche, en utilisant deux échelles, est cette fois-ci relative à la longueur des interfaces ( $L_{0}$ a été choisi égal à 0,25 fois la longueur d'une sous-structure). Elle n'est donc cette fois plus caractérisée par le comportement global de toute la structure et devient une caractéristique de la sous-structuration, [5]. 


\begin{tabular}{|c|c|c|}
\hline Échelle globale -1 processeur & & Échelle fine $-n$ processeurs \\
\hline $\begin{array}{l}\text { Initialisation } \\
\text { réception des } \bar{\sigma}_{d}^{E} \quad \longleftarrow \\
\text { assemblage des contributions } \\
\text { factorisation de }\left[K_{1}\right] \\
\text { montée-descente : problème global } \\
\text { envoi de } \underline{\bar{U}}\end{array}$ & $\longleftarrow$ & $\begin{array}{l}\text { Initialisation } \\
\begin{array}{l}\text { calcul des contributions } \overline{\boldsymbol{\sigma}}_{d}^{E} \\
\text { envoi de } \overline{\boldsymbol{\sigma}}_{d}^{E} \\
\text { initialisation de } \hat{\mathbf{s}} \\
\text { factorisation de }\left[K^{E}\right]+\left[k^{E}\right] \\
\text { réception de } \overline{\underline{U}} \\
\text { calcul du terme de couplage } \boldsymbol{\sigma}_{1}^{E} \\
\text { montée-descente }\end{array}\end{array}$ \\
\hline $\begin{array}{l}\text { Boucle sur les itérations } \\
\text { réception des } \bar{\sigma}_{2 \mid \Omega^{E}} \longleftarrow \\
\text { assemblage des contributions } \\
\text { montée-descente : problème global } \\
\text { envoi de } \underline{\bar{U}}\end{array}$ & $\longrightarrow$ & $\begin{array}{l}\text { Boucle sur les itérations } \\
\begin{array}{|l}\text { calcul du terme de couplage } \overline{\boldsymbol{\sigma}}_{2 \mid \Omega^{E}} \\
\text { envoi de } \overline{\boldsymbol{\sigma}}_{2 \mid \Omega^{E}} \\
\text { étape locale, } \\
\quad \text { indicateur de convergence } \longleftrightarrow \\
\text { réception de } \underline{\bar{U}} \\
\text { calcul du terme de couplage } \boldsymbol{\sigma}_{1}^{E} \\
\text { montée-descente }\end{array}\end{array}$ \\
\hline
\end{tabular}

TABLE 1: Algorithme à deux échelles

\section{Comparaisons}

Avec l'approche proposée ici, on peut remarquer que ni le déplacement, ni les efforts de $\mathbf{s}_{n}$ ne sont continus au travers des interfaces mais que les deux sauts sont améliorés au cours des itérations.

D'autres approches privilégient la continuité du déplacement comme la méthode de Schur primale [16. À chaque itération, elle propose une solution qui vérifie la continuité du déplacement et améliore le déséquilibre en effort avec un algorithme du gradient conjugué sur le problème condensé en déplacement. D'autre part, la méthode de Schur duale, 8, propose une solution qui vérifie l'équilibre aux interfaces et améliore les sauts de déplacement au cours des itérations du gradient conjugué sur le problème condensé en effort. Plus précisément, avec la méthode FETI, la solution courante doit satisfaire à l'équilibre global sur chaque sous-domaine, et le déplacement est alors connu à un mouvement de solide rigide près par sous-domaine. Cela conduit à la résolution d'un problème global sur tous les sous-domaines pour tous ces mouvements de solide rigide. C'est l'analogue du problème à grande échelle.

L'exemple proposé est cette fois-ci une poutre tridimensionnelle avec une charge tranchante parabolique terminale. Deux discrétisations sont proposées, et présentées sur la figure 5] avec des éléments cubiques à 20 nœuds. 
Pour la grande échelle, l'influence de la discrétisation avec des éléments cubiques à 8 nœuds est étudiée comme l'illustre la figure 6 
La figure 7 reporte quant à elle l'erreur en énergie $e_{n}$ en fonction des itérations, pour la méthode de SCHUR primale, sans préconditionnement, pour la méthode FETI sans préconditionnement, puis avec un préconditionneur économique, et enfin le préconditionneur de Dirichlet. Ces trois derniers calculs ont été réalisés par F.-X. Roux sur le PARAGON de l'ONERAChâtillon. L'ancien algorithme LATIN mono-échelle ainsi que l'extension multi-échelles pour les diverses discrétisations de la grande échelle sont aussi reportées. Ils ont été réalisés sur le CRAY-T3D de l'IDRIS à Orsay. Ces deux calculateurs ont été utilisés dans une configuration où 32 processeurs sont disponibles.

Comme des comparaisons en temps entre deux approches dépend des processeurs, réseaux de communication, compilateurs, environnement de programmation, accès disque..., nous avons retenus les principales tendances des algorithmes en pondérant les précédents résultats : après avoir analysé les parties coûteuses des simulations, nous avons identifié les coûts en temps CPU des initialisations des approches FETI et LATIN monoéchelle à 1, en temps CPU équivalent (cumulé sur les 32 processeurs). Après cela, les itérations FETI et LATIN 2-échelles pour le cas (a) sont identifiées en termes de coût. La figure 8 reporte alors l'évolution de l'erreur en fonction de ce temps CPU équivalent.

La même démarche a été adoptée pour la deuxième discrétisation envisagée du problème de référence. Elle conduit cette fois-ci aux évolutions de l'erreur au cours des itérations de la figure 9 et en fonction d'un deuxième temps équivalent sur la figure 10 .

Les caractéristiques des problèmes traités sont reportées dans les tables 2 et 3

\begin{tabular}{|c||c|c|c|c|c|}
\hline \multicolumn{1}{|c||}{ cas } & \multicolumn{5}{c|}{ 1 sous-structure } \\
\cline { 2 - 6 } & $\begin{array}{c}\text { nombre } \\
\text { de nœuds }\end{array}$ & \multirow{2}{*}{$\begin{array}{c}\text { nombre } \\
\text { de ddl }\end{array}$} & $\begin{array}{c}\text { nombre } \\
\text { d'éléments }\end{array}$ & $\begin{array}{c}\text { encombrement (Mo) } \\
\text { (semi-morse) }\end{array}$ & $\begin{array}{c}\text { rélémentaigidités } \\
\text { (semes }\end{array}$ \\
\hline \hline 1 & 425 & 1275 & 64 & 2,1 & 0,96 \\
2 & 1225 & 3675 & 216 & 12,8 & 3,1 \\
\hline
\end{tabular}

TABLE 2: Caractéristiques des problèmes locaux aux sous-structures

Les coûts d'une approche éléments finis directe pour ces deux cas sont en temps CPU équivalent respectif de 6,5 pour le problème à $30051 \mathrm{ddl}$ et 18 pour le problème à $95043 \mathrm{ddl}$ (figure 5 ) : une approche par sousstructuration est d'autant plus efficace que la taille du problème croît. La table 4 reporte ces coûts ainsi que ceux obtenus par une approche multifrontale.

Lors de l'utilisation d'une approche multi-frontale, 4], la condensation coûte plus cher qu'une simple factorisation à cause du remplissage plus 


\begin{tabular}{|c||c|c|c||c|c|c||c|c|c|}
\hline \multirow{2}{*}{ cas } & \multicolumn{3}{c||}{ sous-structuration } & \multicolumn{3}{c||}{ calcul direct } & \multicolumn{3}{c|}{ complément de Schur } \\
\cline { 2 - 10 } & $\begin{array}{c}\text { nb. ddl } \\
\text { total }\end{array}$ & enc. total (Mo) & \begin{tabular}{c} 
nb. \\
\cline { 2 - 9 }
\end{tabular} & $\begin{array}{c}\text { nb. } \\
\text { fact. }\end{array}$ & rig. élém. & $\begin{array}{c}\text { enc. } \\
\text { élém. }\end{array}$ & $\begin{array}{c}\text { nb. } \\
\text { ddl }\end{array}$ & $\begin{array}{c}\text { nb. } \\
\text { nouds }\end{array}$ & $\begin{array}{c}\text { enc. } \\
\text { ddl }\end{array}$ \\
\hline \hline 1 & 40800 & 68,4 & 31 & 2048 & 30051 & 184 & 2977 & 8931 & 68 \\
2 & 117600 & 410 & 102 & 18449 & 95043 & 1252 & 6625 & 19875 & 329 \\
\hline
\end{tabular}

TABLE 3: Caractéristiques des problèmes à résoudre

élevé de la matrice locale de rigidité, afin de traiter en dernier les nouds d'interface. Lorsque, comme ici, le nombre de sous-structures est important, le grand nombre de nœuds sur l'ensemble des interfaces conduit à traiter explicitement un problème condensé (le complément de Schur) de grande taille, ce qui est pénalisant pour ce type d'approche.

De plus, pour l'approche LATIN, le taux de convergence semble peu dépendre de la taille de la maille : il en dépend moins que la version Dirichlet de l'approche FETI, (figures 7 et 9). On peut aussi remarquer de façon générale deux effets dans les approches LATIN 2-échelles lorsque la taille du problème à grande échelle croît : le niveau d'erreur est de plus en plus faible à la première itération car la solution à grande échelle est utilisée pour initier l'algorithme; le taux de convergence est de plus en plus grand, mais comme le coût d'une itération est de plus en plus important, l'ensemble se compense sur les exemples présentés (figures 8 et 10 .

\begin{tabular}{|c|c|c|c|c|}
\hline \multirow[b]{2}{*}{ cas } & \multirow[b]{2}{*}{$\begin{array}{c}\text { direct } \\
\text { (séquentiel) }\end{array}$} & \multicolumn{3}{|c|}{ multi-frontal $(*)$} \\
\hline & & $\begin{array}{c}\text { condensation } \\
\text { + remontée } \\
\text { (parallèle) }\end{array}$ & $\begin{array}{c}\text { résolution } \\
\text { condensée } \\
\text { (séquentiel) }\end{array}$ & total \\
\hline 1 & 6,5 & 2,1 & 2,4 & 4,5 \\
\hline 2 & 18 & 3 & 2,6 & 5,6 \\
\hline
\end{tabular}

(*) avec une renumérotation non optimisée

TABLE 4: Coûts de calcul relatifs suivant la taille du problème

\section{Conclusions}

L'originalité de l'utilisation conjointe d'une approche à grand incrément de temps avec une technique de sous-structuration réside dans le rôle majeur joué par les interfaces qui sont considérées comme des structures particulières avec leurs propres variables et leurs propres équations.

Cela conduit à un algorithme "purement" parallèle qui peut être amélioré par l'introduction d'un schéma à deux échelles. La conséquence est l'émergence 
d'un problème global sur toute la structure à résoudre à chaque itération. L'algorithme en résultant est numériquement extensible; il présente de plus une faible dépendance vis à vis du raffinement du maillage.

Le but à long terme est l'extension à l'analyse de structures à comportement non-linéaire et à grand nombre de degrés de liberté. Les travaux en cours portent sur une version multi-échelles plus proche des techniques d'homogénéisation et qui s'insère complètement dans la trame des méthodes non-incrémentales LATIN.

\section{Remerciements}

Les auteurs tiennent à remercier F.-X. Roux de l'ONERA-Châtillon, pour les comparaisons avec les approches FETI ainsi que l'IDRIS d'Orsay, pour avoir pu accéder au CRAY-T3D.

\section{Références}

[1] J. H. Bramble, J. E. Pasciak, and A. H. Schatz. The construction of preconditioners for elliptic problems by substructuring, I. Math. Comp., 47(175) :103-134, 1986.

[2] L. Champaney, J.-Y. Cognard, D. Dureisseix, and P. Ladevèze. Large scale applications on parallel computers of a mixed domain decomposition method. Computational Mechanics, (19) :253-263, 1997.

[3] J.-Y. Cognard, D. Dureisseix, P. Ladevèze, and P. Lorong. Expérimentation d'une approche parallèle en calcul de structures. Revue Européenne des Éléments Finis, 5(2):197-220, 1996.

[4] I. S. Duff. Parallel implementation of multifrontal schemes. Parallel Computing, 3 :192-204, 1986.

[5] D. Dureisseix. Une approche multi-échelles pour des calculs de structures sur ordinateurs à architecture parallèle. Thèse, ENS de Cachan, Janvier 1997.

[6] D. Dureisseix and P. Ladevèze. Parallel and multi-level strategies for structural analysis. In J.-A. Désidéri, editor, Proceedings of the Second European Conference on Numerical Methods in Engineering, pages 599-604. Wiley, Septembre 1996.

[7] Y. Escaig, G. Touzot, and M. Vayssade. Parallelization of a multilevel domain decomposition method. Computing Systems in Engineering, 5(3) :253-263, 1994.

[8] C. Farhat, J. Mandel, and F.-X. Roux. Optimal convergence properties of the FETI domain decomposition method. Computer Methods in Applied Mechanics and Engineering, 115 :365-385, 1994.

[9] C. Farhat and F.-X. Roux. A method of finite element tearing and interconnecting and its parallel solution algorithm. International Journal for Numerical Methods in Engineering, 32 :1205-1227, 1991. 
[10] C. Farhat and F.-X. Roux. Implicit parallel processing in structural mechanics. In J. T. Oden, editor, Computational Mechanics Advances, volume 2. North-Holland, Juin 1994.

[11] R. Glowinski and P. Le Tallec. Augmented lagrangian interpretation of the nonoverlapping Schwarz alternating method. In T. F. Chan, R. Glowinski, J. Périaux, and O. B. Widlund, editors, Third International Symposium on Domain Decomposition Methods for Partial Differential Equations, pages 224-231, Philadelphia, 1990. SIAM.

[12] W. Hackbush and U. Trottenberg. Multigrid methods, volume 960 of Lecture Notes in Mathematics. Springer Verlag, 1981.

[13] J. Ladevèze. Algorithmes adaptés aux calculs vectoriel et parallèle pour des méthodes de décomposition de domaines. In J. P. Grellier and G. M. Campel, editors, Actes du $3^{e}$ colloque Tendances Actuelles en Calcul de Structures, pages 893-907, Bastia, Novembre 1985. Pluralis.

[14] P. Ladevèze. Mécanique non-linéaire des structures - Nouvelle approche et méthodes de calcul non incrémentales. Hermès, Paris, 1996.

[15] P. Ladevèze and P. Lorong. A large time increment approach with domain decomposition technique for mechanical non linear problems. In R. Glowinski, editor, Comput. Meths. Appl. Sc. Engng., pages 569578, New York, 1992. INRIA, Nova Science.

[16] P. Le Tallec. Domain decomposition methods in computational mechanics. In Computational Mechanics Advances, volume 1. North-Holland, 1994.

[17] P.-L. Lions. On the Schwarz alternating method III : a variant for nonoverlapping subdomains. In T. F. Chan, R. Glowinski, J. Périaux, and O. B. Widlund, editors, Third International Symposium on Domain Decomposition Methods for Partial Differential Equations, pages 202-223, Philadelphia, 1990. SIAM.

[18] J. Mandel. Balancing domain decomposition. Communications in Applied Numerical Methods, 9 :233-241, 1993.

[19] A. K. Noor. New computing systems and future high performance computing environment and their impact on structural analysis and design. Computers $\&$ Structures, 64(1-4) :1-30, 1997.

[20] I. D. Parsons and J. F. Hall. The multigrid method in solid mechanics : part I-algorithm description and behaviour. International Journal for Numerical Methods in Engineering, 29 :719-737, 1990.

[21] B. F. Smith. A domain decomposition algorithm for elliptic problems in three dimensions. Num. Math., 60 :219-234, 1991.

[22] P. Verpeaux, T. Charras, and A. Millard. CASTEM 2000 : une approche moderne du calcul des structures. In J.-M. Fouet, P. Ladevèze, and R. Ohayon, editors, Calcul des Structures et Intelligence Artificielle, volume 2, pages 261-271. Pluralis, 1988. 
[23] G. Yagawa, A. Yoshioka, S. Yoshimura, and N. Soneda. A parallel finite element method with a supercomputer network. Computers 83 Structures, 47 :407-418, 1993.

[24] O. C. Zienkiewicz, J. P. De S. R. Gago, and D. W. Kelly. The hierarchical concept in finite element analysis. Computers 83 Structures, $16(1-4): 53-65,1983$. 


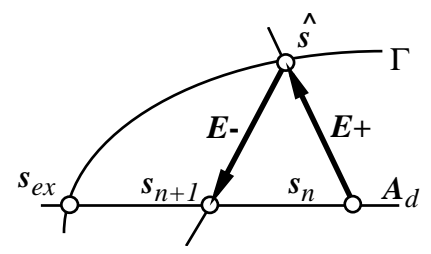

FigurE 2: L'algorithme LATIN à deux étapes

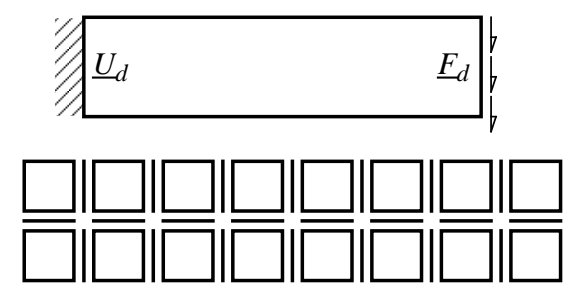

FigurE 3: Problème modèle avec une décomposition en 16 sous-structures et interfaces

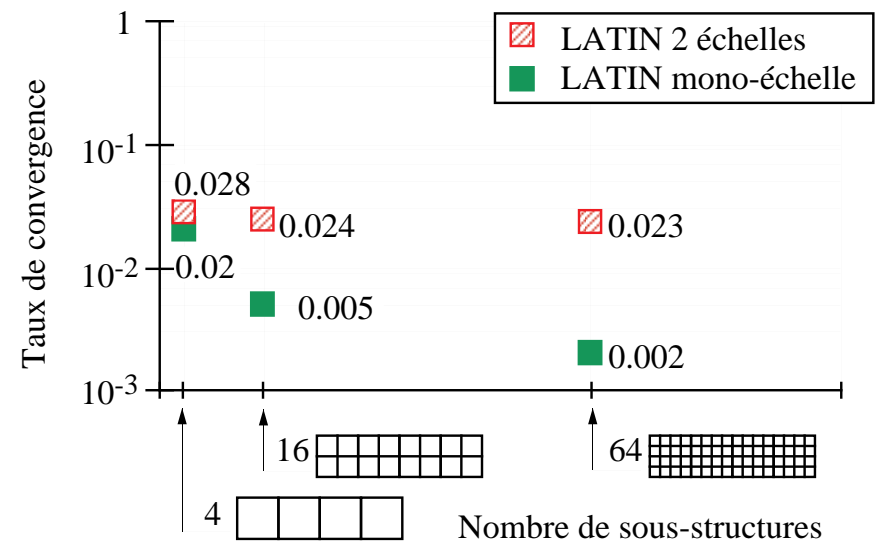

Figure 4: Taux de convergence fonction du nombre de sous-structures 


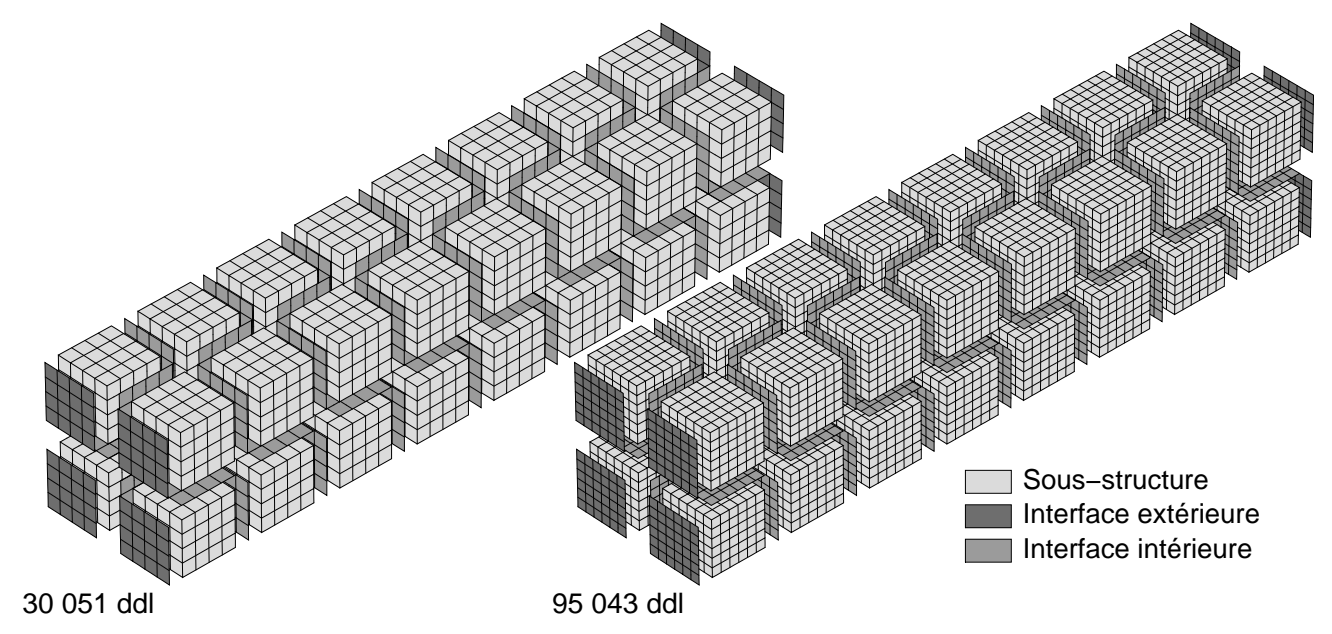

FiguRE 5: Maillages du problème sous-structuré (assemblage de sous-structures et d'interfaces)

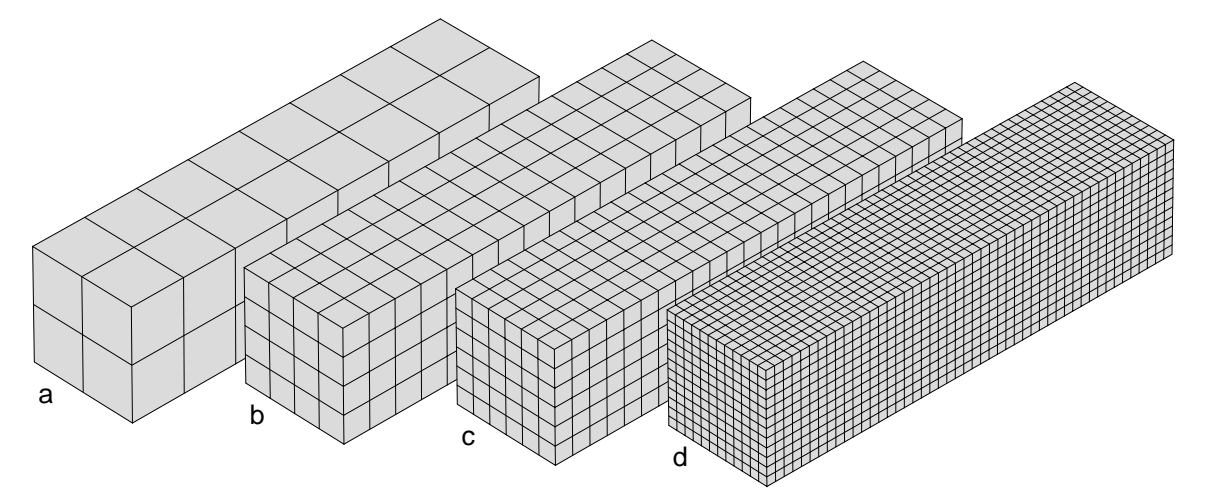

Figure 6: Maillages du problème à grande échelle 


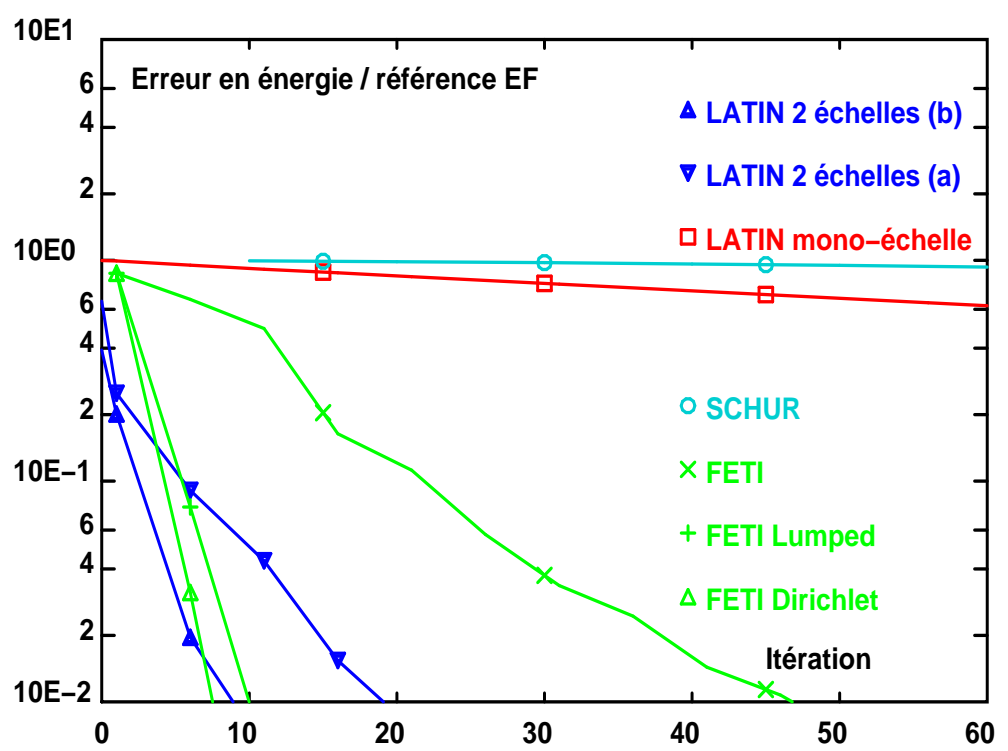

Figure 7: Comparaison des approches pour le premier cas

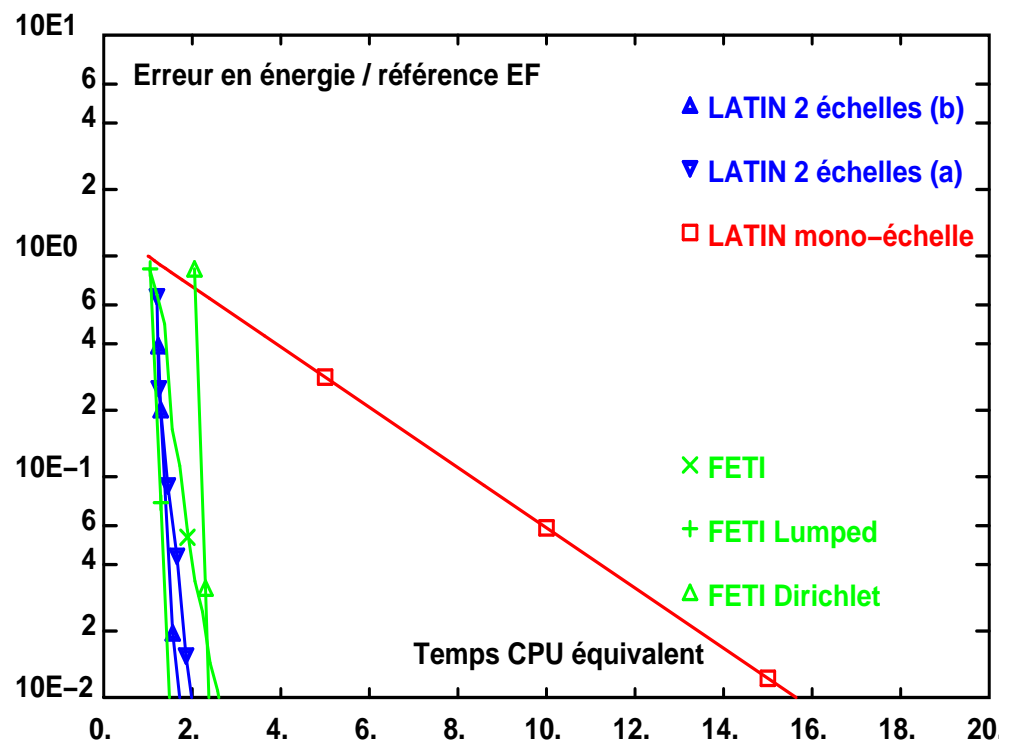

Figure 8: Comparaison des coûts pour le premier cas 


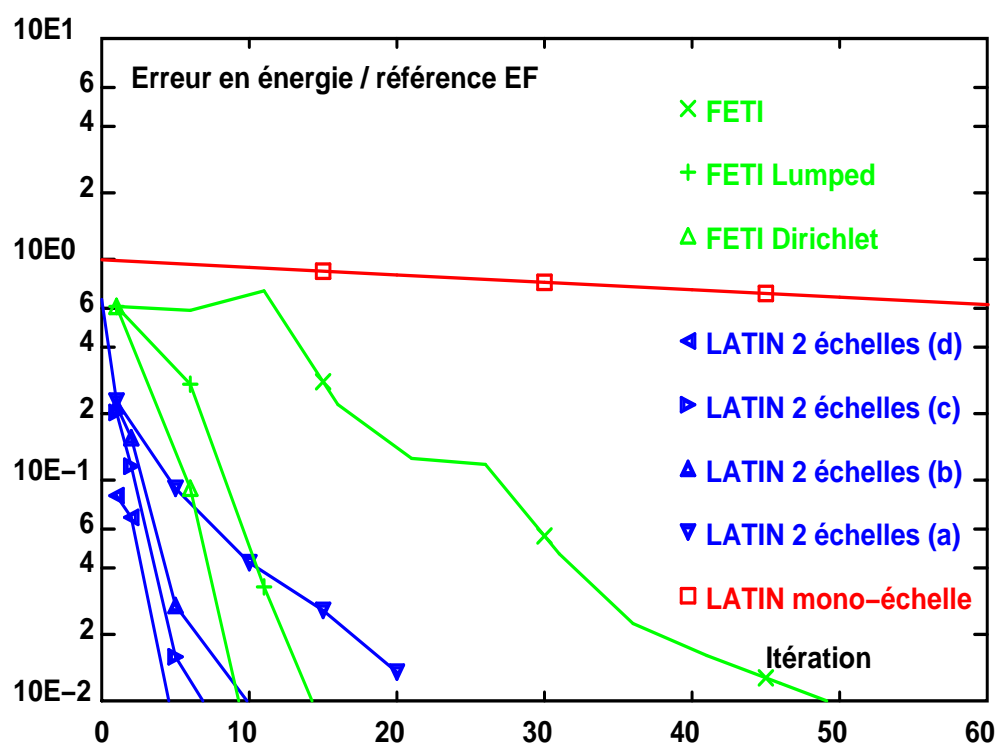

Figure 9: Comparaison des approches pour le second cas

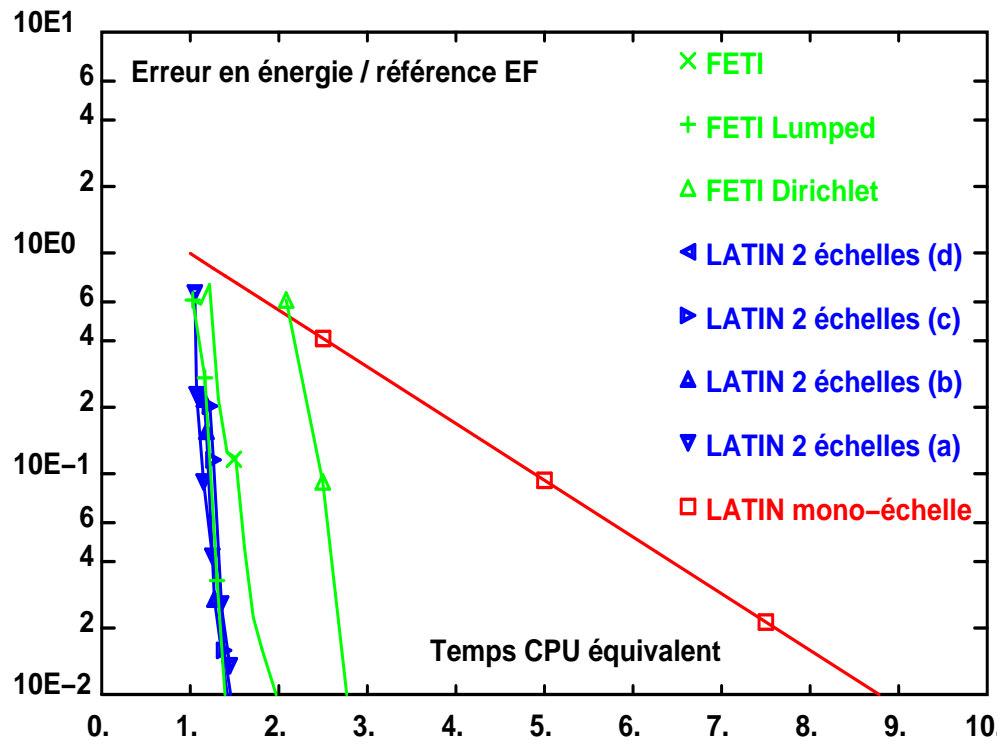

Figure 10: Comparaison des coûts pour le second cas 